\title{
Fuzzy model-based body-wide anatomy recognition in medical images
}

Jayaram K. Udupa a , Dewey Odhner ${ }^{\mathrm{a}}$, Yubing Tong ${ }^{\mathrm{a}}$, Monica M.S. Matsumoto ${ }^{\mathrm{a}}$, Krzysztof C. Ciesielski ${ }^{\mathrm{a}, \mathrm{b}}$, Pavithra Vaideeswaran ${ }^{\mathrm{a}}$, Victoria Ciesielski ${ }^{\mathrm{a}}$, Babak Saboury ${ }^{\mathrm{a}}$, Liming Zhao ${ }^{\mathrm{a}}$, Syedmehrdad Mohammadianrasanani ${ }^{a}$, Drew A. Torigian ${ }^{c}$

${ }^{a}$ Medical Image Processing Group, ${ }^{c}$ Dept. of Radiology, University of Pennsylvania, 423 Guardian

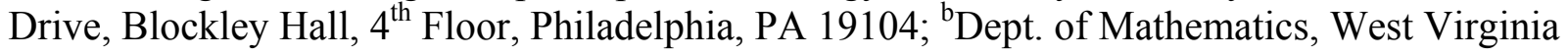
University, Morgantown, WV.

\begin{abstract}
To make Quantitative Radiology a reality in routine radiological practice, computerized automatic anatomy recognition (AAR) becomes essential. Previously, we presented a fuzzy object modeling strategy for AAR. This paper presents several advances in this project including streamlined definition of open-ended anatomic objects, extension to multiple imaging modalities, and demonstration of the same AAR approach on multiple body regions. The AAR approach consists of the following steps: (a) Collecting image data for each population group $G$ and body region $B$. (b) Delineating in these images the objects in $B$ to be modeled. (c) Building Fuzzy Object Models (FOMs) for $B$. (d) Recognizing individual objects in a given image of $B$ by using the models. (e) Delineating the recognized objects. (f) Implementing the computationally intensive steps in a graphics processing unit (GPU).

Image data are collected for $B$ and $G$ from our existing patient image database. Fuzzy models for the individual objects are built and assembled into a model of $B$ as per a chosen hierarchy of the objects in $B$. A global recognition strategy is used to determine the pose of the objects within a given image $I$ following the hierarchy. The recognized pose is utilized to delineate the objects, also hierarchically. Based on three body regions tested utilizing both computed tomography (CT) and magnetic resonance (MR) imagery, recognition accuracy for non-sparse objects has been found to be generally sufficient ( 3 to $11 \mathrm{~mm}$ or $2-3$ voxels) to yield delineation false positive (FP) and true positive (TP) values of $<5 \%$ and $\geq$ $90 \%$, respectively. The sparse objects require further work to improve their recognition accuracy.
\end{abstract}

Keywords: Shape modeling, fuzzy models, object recognition, segmentation, graph cut, and fuzzy connectedness.

\section{INTRODUCTION}

It is widely recognized that quantitative radiology $(\mathrm{QR})$ in routine clinical practice can lead to improved sensitivity, specificity, accuracy, and precision of early diagnosis; objective and standardized treatment response assessment; improved understanding of "normal"; increased ease of disease measurement and reporting; discovery of new disease biomarkers; better outcome assessment; effective handling of the large volume of image information; and effective combined utilization of multiple modalities. AAR becomes essential to facilitate QR. AAR can also help computer-aided detection (CAD) systems as a first processing step and to reduce false positives.

Anatomy recognition methods to date [1 - 4] have taken two strategies - (i) determining the whereabouts of an object in the form of a rectangular region of interest (ROI), and (ii) determining the pose of an object model in the image with the intent of subsequently delineating the object. Most of the focus has been on specific organs/ organ systems and not on the same general framework operating on a multitude of organs body-wide, especially for the latter strategies. All reported modeling strategies have a statistical framework, none taking a fuzzy approach, except [5-7]. Fuzzy set concepts have been used extensively otherwise in image processing and 3D visualization. Fuzzy modeling approaches allow bringing anatomic information in an all-digital form into graph theoretic frameworks, obviating the need for continuous assumptions made otherwise about shapes, random phenomenon etc. They also allow capturing information about uncertainties at the patient level (e.g., blur, partial volume effects) and at the group $(G)$ level and codifying this information within the model for $B$. We model the hierarchical arrangement inherent in the anatomic layout and exploit this codification to make AAR effective and efficient.

Medical Imaging 2013: Image-Guided Procedures, Robotic Interventions, and Modeling,

edited by David R. Holmes III, Ziv R. Yaniv, Proc. of SPIE Vol. 8671, 86712B

(C) 2013 SPIE ·CCC code: $1605-7422 / 13 / \$ 18 \cdot$ doi: $10.1117 / 12.2007983$ 
Section 2 presents the up-to-date AAR methodology including a new recognition strategy based on thresholding that is more accurate and faster than our previous strategies. Section 3 illustrates the method on different body regions. Section 4 states our conclusions.

\section{THE AAR METHODOLOGY}

The AAR approach consists of the following six steps: 1. Collecting image data for $B$ and $G$. 2. Delineating objects for modeling. 3. Building fuzzy models of objects. 4. Recognizing objects of $B$ in a given image $I$ of $B$ for $G$. 5. Delineating the recognized objects. 6. Implementing the computationally intensive steps in GPU. These steps are described in some detail below.

The body is divided into body regions $B_{1}, \ldots, B_{r}$. Models are built for each specific population group $G$ (whatever way $G$ is defined). Below, $G$ and the body region $B$ are considered as variables.

1. Collecting image data for $B$ and $G$ : Our goal is to build models for normal anatomy. Images that are radiologically normal for $B$ and $G$ are selected from our patient database.

2. Object definition for modeling: Each body region is defined consistently in terms of a starting and ending anatomic location. For axial slice data, these locations are determined in terms of transverse positions. For other slice orientations, similar definitions apply. Similarly each object included in $B$ is defined properly irrespective of whether it is open-ended (crossing body regions) or closed (but contiguous with other objects). Without such operational definitions, modeling becomes ambiguous and meaningless.

Once their definitions are created, the objects are delineated for the purpose of building a model of $B$ for $G$ by a combination of methods based on live wire, thresholding, and manual painting, tracing and correction, adhering to the definition. To minimize human labor, algorithms in terms of a proper combination of these methods and the order in which objects are delineated are devised first.

3. Building Fuzzy Object Models (FOMs): The Fuzzy Object Model FOM(B) for $B$ (and $G$ ) is a quintuple, $F O M(B)=$ $[H, M, \rho, \lambda, \eta] . H$ here is a hierarchy, represented as a tree, of the objects $O_{1}, \ldots, O_{L}$ in $B$ which are considered for inclusion in model building. While each $B$ has its own hierarchy, the $B$ 's form the offspring of the root which denotes the whole body $W B$ (see Figure 1). We have studied three body regions so far - neck, thorax, and the abdomen. The hierarchy for thorax is shown in Figure 1 as an example.

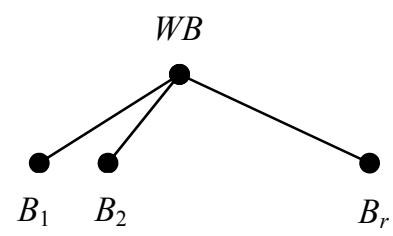

Figure 1. Left: Hierarchy for whole body. Right: Hierarchy for Thorax. RS: Respiratory System; TS: Thoracic Skeleton; IMS: Internal Mediastinum; RPS, LPS: Right \& Left Pleural Space; TB: Trachea \& Bronchi; E: Esophagus; PC: Pericardium; AS, VS: Arterial \& Venous Systems.

$\mathrm{M}=\left\{F M\left(\mathrm{O}_{\ell}\right): 1 \leq \ell \leq L\right\}$ is a set of fuzzy models, one model per object. $\rho$ describes the parent-to-offspring relationship in $H$ over $G: \rho=\left\{\rho_{\ell, k}: O_{\ell}\right.$ is a parent of $\left.O_{k}, 1 \leq \ell, k \leq L\right\}$. It also encodes $W B$ to body region relationships. $\lambda$ is a set of scale factor ranges $\lambda=\left\{\lambda_{\ell}=\left[\lambda_{\ell}^{b}, \lambda_{\ell}^{h}\right]: 1 \leq \ell \leq L\right\}$ indicating the size variation of each object $O_{\ell}$ over $G$. $\eta$ represents a set of measurements pertaining to the objects in $B$. For details, see [7].

4. Object recognition: The task of AAR is broken up into two components - Recognition and delineation. The goal of recognition is to determine where objects $O_{\ell}$ are in a given test image $I$, meaning, to determine the pose of $F M\left(\mathrm{O}_{\ell}\right)$ in $I, 1$ $\leq \ell \leq L$. The goal of delineation is to mark the precise spatial occupation of the objects in $I$. 
There is an initial global recognition step whose goal is an initial placement of $F O M(B)$ in $I$ in close proximity to the actual objects in $I$. This process proceeds following the hierarchy $H$. The descendants $O_{k}$ are recognized by a knowledge of the pose of their already recognized parent $O_{\ell}$ and of the parent-to-offspring relationship $\rho_{\ell, k}$. We have developed and evaluated the following four strategies for recognition.

One-shot: The root object (skin boundary) is identified first by applying a threshold to $I$ first and then placing the model at a pose corresponding to a relationship between the resulting binary image and the b-scale image of $I$ [8]. This relationship is learned from the training images. The pose of all other objects are adjusted accordingly hierarchically by using $\rho_{\ell, k}$.

Fisher Linear Discriminant (FLD): This method [9] uses certain boundary features and a trained FLD to search for an optimum pose for all objects (other than the root) in a hierarchical manner.

B-scale: This method [9] is similar to FLD except that it uses the b-scale to minimize a boundary cost function akin to live wire.

Thresholded optimum search: This method also works hierarchically but uses known (learned) fixed object threshold interval and minimal false positive and false negative of the thresholded image with respect to $F M\left(O_{\ell}\right)$ for best recognition. In the case of MR images, intensity standardization is essential for this method to work effectively.

5. Object delineation: We have implemented Graph Cut (GC) and Iterative Relative Fuzzy Connectedness (IRFC) algorithms $[10,11]$. Both have a model component in their energy function. The seeds are found based on $F M\left(O_{\ell}\right)$ which results from recognition. Object and background seeds are found automatically as voxels in $I$ that satisfy an object and model threshold criterion.

6. Implementation in GPU: Key computationally intensive operations in our AAR methodology are image interpolation, distance transform on binary images, and graph-based delineation. We have developed a GPU implementation of the linear time IRFC algorithm [12] and a parallel version of a distance transform algorithm [13]. GPU implementation of the latter and interpolation operation are underway.

\section{EXPERIMENTAL RESULTS}

We have experimented with three body regions - thorax, abdomen, and neck (upper airway and surrounding organs) with 11, 11, and 17 objects, respectively, where each region has its own hierarchy. For thorax and abdomen, $G$ constitutes male subjects 50-60 years of age and the images were contrast-enhanced CT $\left(512 \times 512 \times 80,0.9 \times 0.9 \times 5 \mathrm{~mm}^{3}\right)$ from 41 and 48 patients, respectively. Data from roughly half of the population were used for model building and from the rest for testing. For the thorax, the objects included are as in Figure 1. For the abdomen, the objects included are: skin (outer) boundary, abdominal skeleton, soft tissues, liver, kidneys, spleen, subcutaneous adipose tissue, muscle, abdominal aorta, and inferior vena cava. For the neck: skin boundary, soft tissues, air and bone, fat pads, mandible, airways (nasal, pharyngeal, laryngeal), tongue, palate (hard and soft), adenoid, and tonsils. For the neck, modeling and testing were done based on MR images of twelve 8 to 17 year-old normal female subjects. A 10-fold testing was carried out based on data from 5 additional subjects. Both axial and sagittal T1-weighted acquisitions were used for this last group. Most of the objects for neck were modeled from axial data but some were modeled from sagittal data. A composite $\operatorname{FOM}(B)$ was created from the two types of image data.

Fuzzy models of objects in different combinations are shown in Figure 2 for the three body regions. The size and geographic layout of objects have interesting relationships over $G$ which can be exploited in model building and recognition [14]. For example, the sizes of bilateral organs are strongly correlated, and some organ sizes are strongly correlated with distances among them. Some recognition results are illustrated in Figure 3 where the adjusted (at recognition) model is overlaid on the test images. In all body regions, the ratio of estimated to true object size is excellent $(\sim 1)$. Average recognition positional error (in $\mathrm{mm}$ ) is shown in Table 1 separately for sparse (thin, tubular) objects and non-sparse objects for the best method (Optimal Threshold) over the tested images and objects. We have 
observed that a typical recognition accuracy of 10-12 mm or better (i.e., 2-3 voxels or better) in our AAR system yields delineation results with TP $>90 \%$ and FP $<5 \%$. See References $[15,16,17]$ which show delineation results for specific body regions and applications obtained by using the same AAR system.

Table 1. Mean recognition positional accuracy (in $\mathrm{mm}$ ) for the different body regions and types of objects.

\begin{tabular}{c|ccc}
\hline Objects & Thorax & Abdomen & Neck \\
\hline Non-Sparse & 6.5 & 11.3 & 3.4 \\
Sparse & 24.6 & 16.8 & 5.0 \\
\hline
\end{tabular}

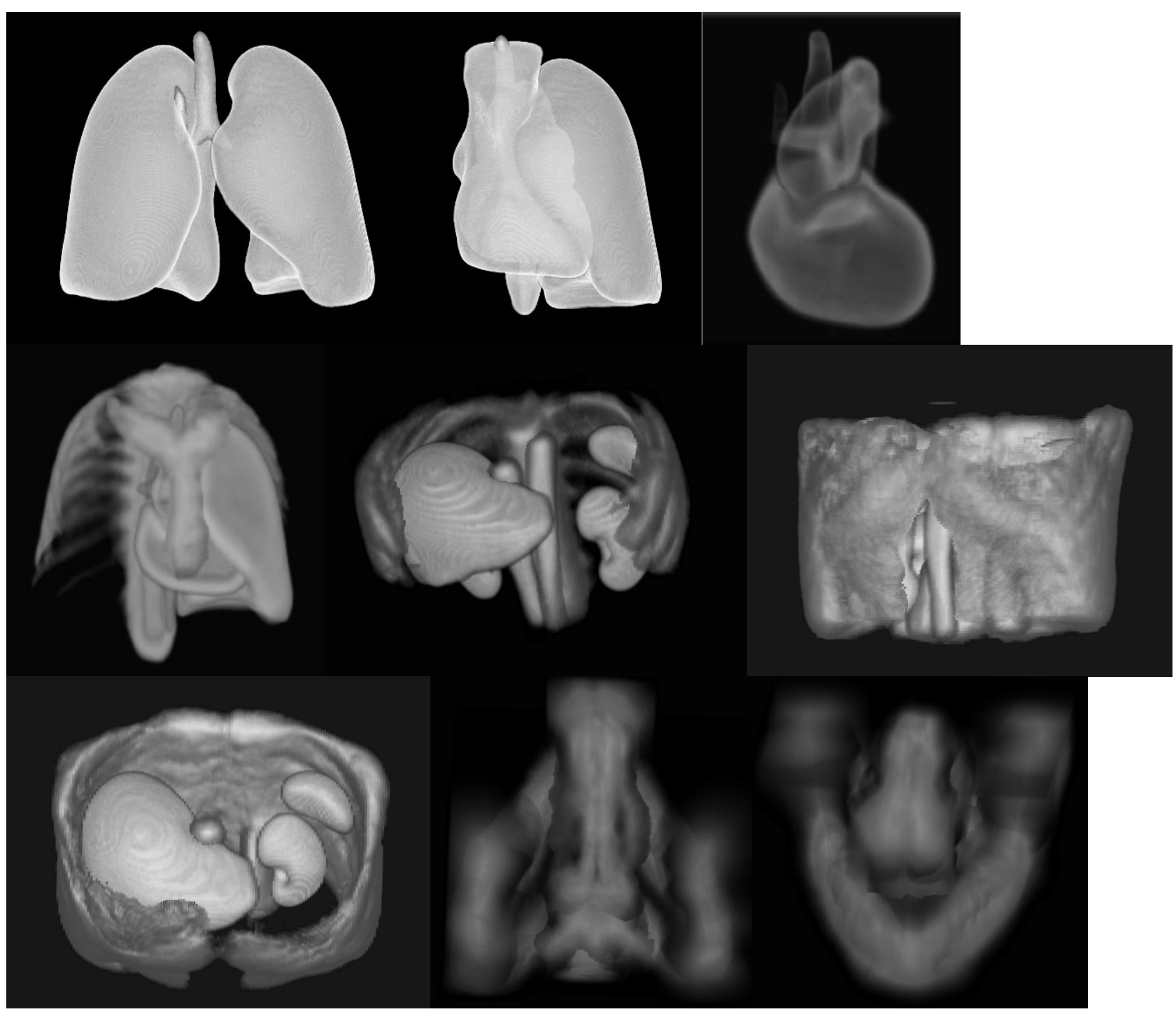

Figure 2. Examples of fuzzy models from the three body regions in different combinations of the objects are shown in volume rendering. 


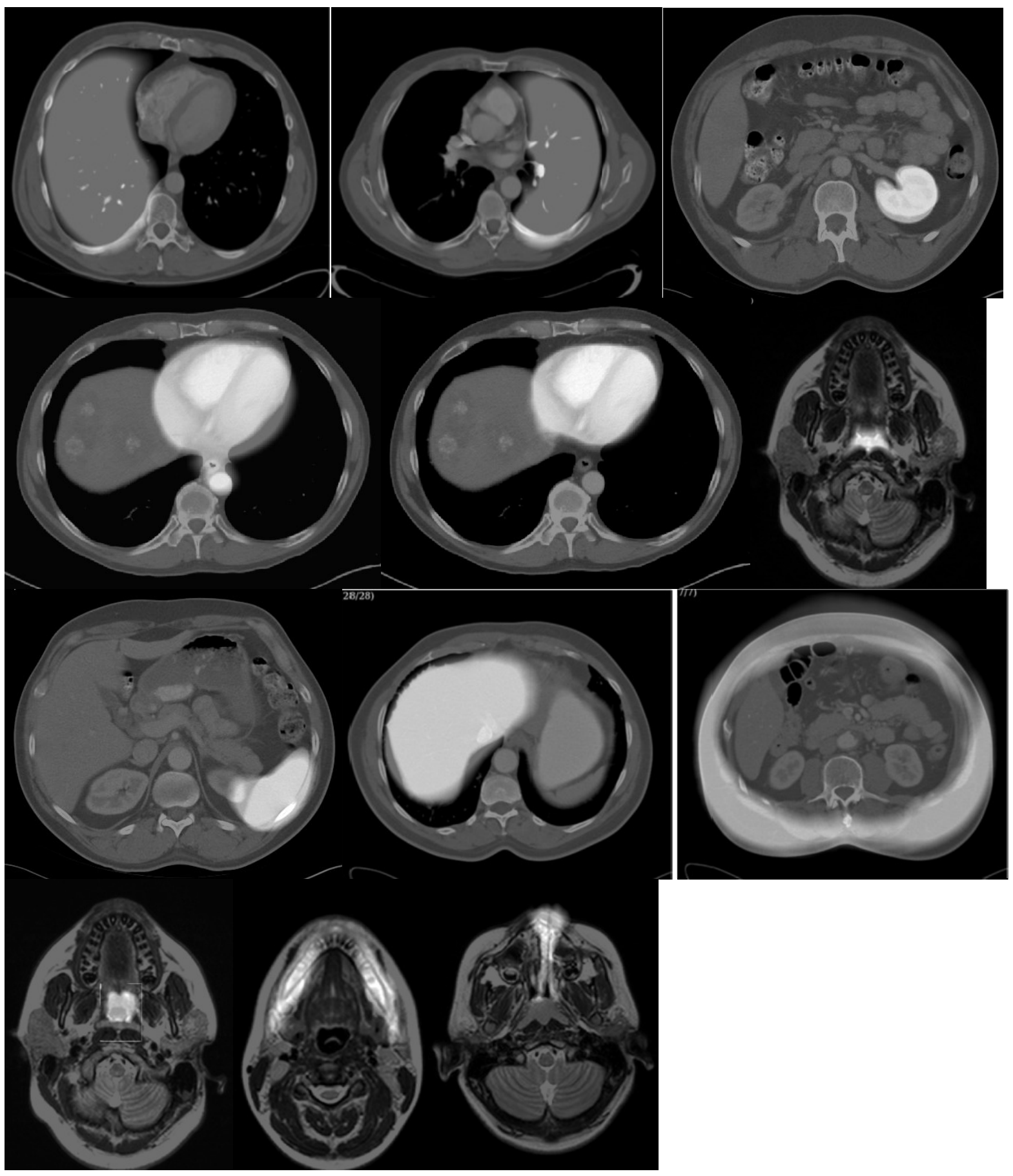

Figure 3. Fuzzy models at recognition overlaid on test image slices. The models appear as a bright cloud over slice display. L to R, Top to Bottom: RPS, LPS, left kidney, IMS, PC, adenoid, spleen, liver, subcutaneous adipose tissue, airway, mandible, and airway (nasal). Note that the overlay appears dimmer in the case of RPS and LPS because of the actual dark appearance of those objects. 


\section{CONCLUDING REMARKS}

It is feasible to design a general AAR system that can work in different body regions and anatomic image modalities. For body-wide AAR, this is perhaps needed to make such a system manageable. In this paper, we have taken a fuzzy approach to modeling.

Positional recognition accuracy for non-sparse and some sparse objects is good enough for delineation TP and FP of $\geq 90 \%$ and $<0.5 \%$. See References [15-17] for delineation results. We note that for Thorax and Abdomen, our data sets have $5 \mathrm{~mm}$ slice spacing, which means that for non-sparse objects recognition accuracy (in position) is within about 2-3 voxels. For the neck region, where slice spacing is smaller, the absolute error is generally smaller (see [16]) and still about 2-3 voxels.

For several sparse objects such as vessels (in Thorax and Abdomen), recognition needs improvement. We observed that modeling based on exact binary object shapes given as training data sets is not effective for some sparse objects. We are exploring ideas of rough sets for this purpose.

Our main focus has been object recognition and the related aspects of model building. Without effective recognition, delineation would fail. Once done effectively, the AAR methodology can be applied to different modalities and body regions with minor changes [15-17].

\section{Acknowledgements}

This research work is supported partly by a DHHS grant HL 105212.

\section{REFERENCES}

[1] Criminisi, A., Shotton, J., and Bucciarelli, S., "Decision Forests with Long-Range Spatial Context for Organ Localization in CT Volumes," MICCAI workshop on Probabilistic Models for Medical Image Analysis (MICCAIPMMIA), 2009.

[2] Zhou, X., Yamaguchi, S., Zhou, X., Chen, H., Hara, T., Yokoyama, R., Kanematsu, M., and Fujita, H., “Automatic organ localizations on 3D CT images by using majority-voting of multiple 2D detections based on local binary patterns and Haar-like features, Proc. SPIE, 8676, (2013).

[3] Deserno, T.M., (editor) Recent Advances in Biomedical Image Processing and Analysis, Springer, Hiedelberg, Germany, Chapter 5 (2011).

[4] Lotjonen, J.M.P., Wolz, R., Koikkalainen, J.R., Thurfjel, L., Waldemar, G., Soininen, H., Rueckert, D., "Fast and robust multi-atlas segmentation of brain magnetic resonance images," Neuroimaging, 49, 2352-2365 (2010).

[5] Miranda, P.A.V., Falcao, A.X., Udupa J.K., "Clouds: A model for synergistic image segmentation, Proceedings of the ISBI, 209-212 (2008).

[6] Miranda, P.A.V., Falcao, A.X., Udupa J.K., "Cloud Bank: A multiple clouds model and its use in MR brain image segmentation," Proc. ISBI, 506-509 (2009).

[7] Udupa, J.K., Odhner, D., Falcao, A.X., Ciesielski, K.C., Miranda, P.A.V., Vaideeswaran, P., Mishra, S., Grevera G.J., Saboury, B., Torigian, D.A., "Fuzzy object modeling," Proc. SPIE 7964:79640B-1-10 (2011).

[8] Bagci, U., Udupa, J.K., Chen, X., Torigian, D.A., "Model-based multi-object recognition of anatomical structures in medical images," IEEE Transactions on Medical Imaging, 31, 777-789 (2012).

[9] Udupa, J.K., Odhner, D., Matsumoto, M.M.S., Falcão, A.X., Miranda, P.A.V., Ciesielski, K.C., Grevera G.J., Saboury, B., Torigian, D.A., "Automatic anatomy recognition via fuzzy object models," Proc. SPIE, 8316, 831605-1 - 831605-8 (2012).

[10] Boykov, Y., Kolmogorov, V., "An experimental comparison of min-cut/max-flow algorithms for energy minimization in vision," IEEE Pattern Analysis \& Machine Intelligence, 26, 1124 - 1137 (2004). 
[11] Ciesielski, K.C., Udupa, J.K., Falcao, A.X., Miranda, P.A.V. , "Fuzzy connectedness image segmentation in graph cut formulation: a linear time algorithm and a comparative analysis," Journal of Mathematical Imaging and Vision, 44, 375-398 (2012).

[12] Zhuge, Y., Udupa J.K., Ciesielski, K.C., Falcao, A.X., Miranda, P.A.V., "GPU based iterative relative fuzzy connectedness image segmentation," Proc. SPIE, 8316, 04-1 - 04-11 (2012).

[13] Ciesielski, K.C., Chen, X., Udupa, J.K., Grevera, G.J., "Linear time algorithms for exact distance transform," Journal of Mathematical Imaging and Vision, 39, 193-209 (2011).

[14] Matsumoto, M.M.S., Udupa, J.K., "Optimal hierarchy for fuzzy object models," Proc. SPIE, 8671 (2013).

[15] Ciesielski, K.C., Udupa, J.K., Odhner, D., Zhao, L., "Fuzzy model based object delineation via energy minimization," Proc. SPIE, 8669 (2013).

[16] Tong, Y., Udupa J.K., Odhner, D., Sin, S., Arens, R., "Recognition of Upper Airway and Surrounding Structures at MRI in Pediatric PCOS and OSAS," Proc. SPIE, 8670, (2013).

[17] Tong, Y., Udupa J.K., Odhner, D., Sin, S., Arens, R., "Abdominal Adiposity Quantification at MRI via Fuzzy Model-Based Anatomy Recognition,” Proc. SPIE, 8672, (2013). 